Case Report Journal of Epilepsy Research pISSN 2233-6249 / elSSN 2233-625

Received May 23, 2017

Accepted September 25, 2017

Corresponding author: Hussein Algahtani Division of Neurology, Department of Medicine, King Abdulaziz Medical City, King Saud bin Abdulaziz University for Health Sciences, P.O. Box 12723, Jeddah 21483, Saudi Arabia

Tel. +966-556633130

E-mail; halgahtani@hotmail.com

\section{Levetiracetam-Induced Skin Hyperpigmentation: An Extremely Rare Undesirable Side Effect}

\author{
Hussein Algahtani ${ }^{1}$, Siham Marghalani ${ }^{1}$, Mohamed Satti ${ }^{1}$, Bader Shirah ${ }^{2}$ \\ ${ }^{1}$ Division of Neurology, Department of Medicine, King Abdulaziz Medical City, King Saud bin Abdulaziz University \\ for Health Sciences, Jeddah; ${ }^{2}$ King Abdullah International Medical Research Center, King Saud bin Abdulaziz \\ University for Health Sciences, Jeddah, Saudi Arabia
}

\begin{abstract}
Levetiracetam is one of the newer second-generation antiepileptic drugs with multiple mechanisms of action. Cutaneous side effects due to levetiracetam are rarely reported in the literature. In this article, we describe a patient with skin hyperpigmentation due to the treatment with levetiracetam with complete resolution after discontinuation of the medication. In addition, we review the topic and hypothesize the mechanism behind this rare complication. To the best of our knowledge, this is the first report of skin hyperpigmentation as a side effect of levetiracetam in the literature. The prescribing physicians should inform the patients about all potential side effect of levetiracetam including skin hyperpigmentation. Similar to many undiagnosed conditions, increased awareness of their existence is the key to diagnosis. Early recognition and timely cessation of therapy are important to reverse this effect. Further studies should be conducted to explore the pathophysiology of this rare side effect. (2017;7:106-108)
\end{abstract}

Key words: Levetiracetam, Skin hyperpigmentation, Adverse effect, Drug-induced

\section{Introduction}

Levetiracetam is one of the newer second-generation antiepileptic drugs with multiple mechanisms of action. It is a broad-spectrum antiepileptic drug that was approved by the United States Food and Drug Administration in 1999 and became one of the most commonly prescribed medications for the treatment of partial and generalized epilepsy (first choice and add-on).' Levetiracetam has a favorable adverse effects profile with the 5 most common adverse effects being nasopharyngitis, somnolence, asthenia/fatigue, dizziness, and nervousness/irritability. Its minimal drug interactions, few side effects, and broad-spectrum efficacy contributed to its wide use for the treatment of seizures. ${ }^{2}$ Skin hyperpigmentation is a common side effect of a variety of drugs such as beta-blockers and non-steroidal anti-inflammatory drugs. However, it was never reported in the literature as an adverse effect of levetiracetam. ${ }^{3}$ In this article, we describe a patient with skin hyperpigmentation due to the treatment with levetiracetam with complete resolution after discontinuation of the medication. In addition, we review the topic and hypothesize the mechanism behind this rare complication.

\section{Case}

A 54-year-old female, known case of epilepsy (partial, complex partial with secondary generalization) following herpes simplex virus encephalitis two years ago. She was started on carbamazepine which was gradually increased to a dose of 1,200 mg per day without complete control of seizure frequency. One-year ago, levetiracetam was added to achieve a better control with success and complete remission of her seizures. She was kept on 1,000 mg of levetiracetam twice daily. She noticed a change in the color of her skin that started diffusely six months following the addition of levetiracetam. Her skin became dark all over her body including face, arms, trunk, and legs. To confirm this claim, old photos taken over many years prior to the initiation of this medication were reviewed with a significant change in the color of her skin (Fig. 1). She was not on any medication that can cause hyperpigmentation such as oral contraceptives or non-steroidal anti-inflammatory drugs. This complaint interfered to some degree with her social and marital relationships. Her past medical, family, and medication history were unremarkable. Her examination showed generalized hyperpigmentation with Fitzpatrick skin type IV. Examination of mucous membranes, nails, and retina was unremarkable. A skin biopsy showed mild epidermal acanthosis (an 


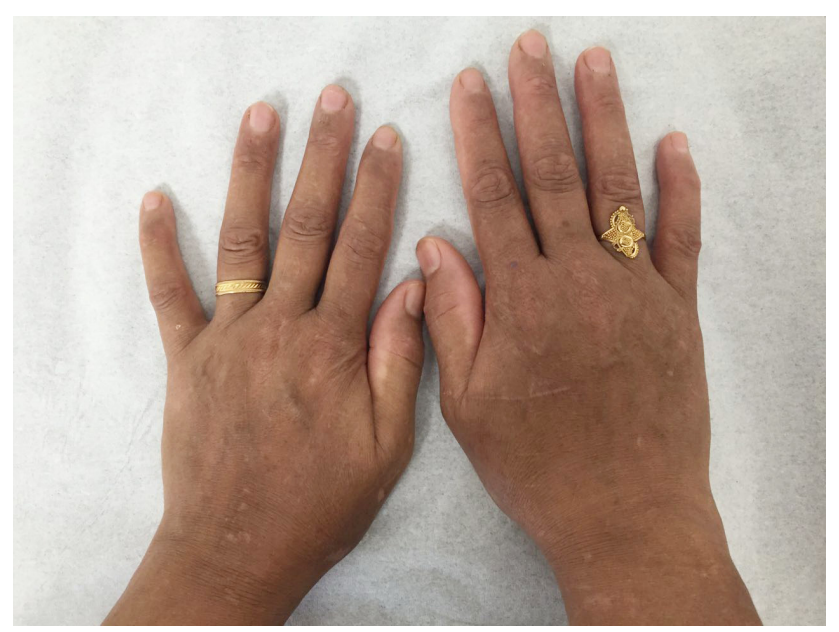

Figure 1. The patient's image showing features of hyperpigmentation.

increase in the thickness of the stratum spinosum of the epidermis) with hyperpigmentation of the basal layer. In addition, there was upper dermal fibroplasia and pigmentary incontinence (Fig. 2). Work up for systemic disorders causing hyperpigmentation including thyroid function tests, electrolytes, serum cortisol, and renal function tests were unremarkable. Modification of the treatment was done with the addition of pregabalin in gradually increasing doses and gradual decrease in levetiracetam dose until complete discontinuation. In a follow-up visit to the clinic six months later, her skin hyperpigmentation improved significantly, and the patient was satisfied and continued to be seizure free.

\section{Discussion}

Melanogenesis is the process of converting tyrosine, which is produced in the liver, to melanin. The color of the skin is determined by the amount of melanin pigment that is produced by this reaction within the melanocytes, which are found in the basal layer of the epidermis. ${ }^{4}$ Melanocyte-stimulating hormone is produced by the anterior pituitary gland and is responsible for stimulating the formation of the melanin and its dissemination to the epidermis. Adrenocorticotropic hormone is another hormone produced by the pituitary gland with a melanocyte-stimulating hormone sequence. It is more abundant and is physiologically more important than melanocyte-stimulating hormone in determining the amount of melanin in the skin. ${ }^{5}$ The three basic types of melanin are eumelanin, pheomelanin, and neuromelanin. The most abundant type is eumelanin, which is responsible for the brown and black pigmentation of human skin. ${ }^{6}$

Fitzpatrick skin typing test is a classification scale for the color of
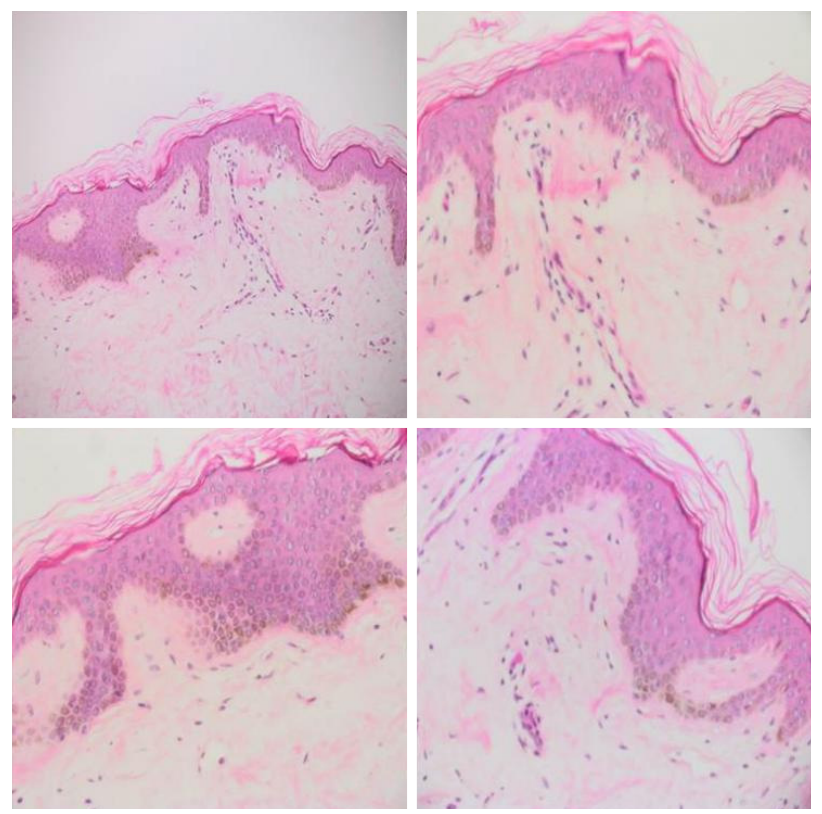

Figure 2. Hematoxylin and eosin section showing melanin hyperpigmentation of basal keratinocytes and upper dermal pigmentary incontinence with many scattered melanophages (the upper two images: $\times 100$; the lower two images: $\times 400$ ).

the skin that estimates the response to ultraviolet light by different skin types. It is composed of six categories ranging from pale white (type I) to deeply pigmented dark brown or black (type VI). Our patient had Fitzpatrick skin type IV. This type appears light brown that burns minimally and always tans well. Fitzpatrick skin type IV may be prone to an overactive production of melanin after exposure to sunlight or receiving hormonal therapy, which may be an additional factor behind the development of hyperpigmentation as a side effect of levetiracetam. ${ }^{4,6}$

Hyperpigmentation is an acquired darkening of the skin, mucous membranes, or nails that may result from sun damage, infections, autoimmune diseases, contact dermatitis, photosensitivity, or allergic reactions. ${ }^{3}$ Medications are the cause of skin hyperpigmentation in around $10-20 \%$ of the patients with the classic triggers being chemotherapeutics, oral contraceptives, non-steroidal anti-inflammatory drugs, and a variety of drugs such as amiodarone and chloroquine. ${ }^{7}$ The level of evidence supporting a cause relationship between hyperpigmentation and medication use is mainly derived from case reports with systematic reviews being rarely found in the literature. ${ }^{3}$

The pathogenesis of drug-induced hyperpigmentation is unknown in some cases. However, the accumulation of melanin in the dermis instead of the basal layer of the epidermis is considered the end results in most of the cases. The accumulation of melanin may result 
from overproduction by epidermal melanocytes that are stimulated by the drug (melanocytic hypermelanosis). It may also represent a nonspecific cutaneous inflammatory reaction to the medication. Another possibility is the formation a stable drug-melanin complex which prevents melanin clearance in the dermal macrophages. Another mechanism may be the accumulation of the triggering medication without melanin. Synthesis of other pigments such as lipofuscin under the direct influence of the drug with subsequent deposition may cause hyperpigmentation. Additionally, the deposition of iron as a result of drug-induced damage to dermal vessels may lead to hyperpigmentation. $3,7,8$

Cutaneous side effects are commonly associated with antiepileptic drugs such as carbamazepine, phenobarbitone, phenytoin, and lamotrigine. These side effects may be mild such as maculopapular rash or more severe and life-threatening such as drug reaction eosinophilia and systemic symptoms. These cutaneous manifestations may appear within a few hours to weeks of the initiation of the antiepileptic drug. Cutaneous side effects due to levetiracetam are rarely reported in the literature. They include angioedema, Stevens Johnson syndrome, drug reaction eosinophilia and systemic symptoms, toxic epidermal necrolysis, hair loss, morbilliform rash, and maculopapular exanthema. ${ }^{9}$ To the best of our knowledge, this is the first report of skin hyperpigmentation as a side effect of levetiracetam in the literature. Although our patient was also on carbamazepine, we speculate that the hyperpigmentation was due to levetiracetam since she was on carbamazepine for one year without the occurrence of hyperpigmentation, and she developed this side effect six months after the addition of levetiracetam. In addition, the hyperpigmentation was reversed by the cessation of levetiracetam while she was still on carbamazepine making levetiracetam the most likely offending drug.

A skin biopsy is important to identify the histological features of the affected skin. The treatment of drug-induced skin hyperpigmentation consists of interruption of the offending drug and avoidance of sun exposure. In most of the patients, a complete resolution is often achieved following these measures. However, the hyperpigmentation may persist for a long period of time or may become permanent in a small number of patients. ${ }^{10}$
The present case illustrates that skin hyperpigmentation may occur as an adverse effect of levetiracetam. The prescribing physicians should inform the patients about all potential side effect of levetiracetam including skin hyperpigmentation. Similar to many undiagnosed conditions, increased awareness of their existence is the key to diagnosis. Early recognition and timely cessation of therapy are important to reverse this effect. Further studies should be conducted to explore the pathophysiology of this rare side effect.

\section{Conflict of Interest}

The authors declare that they have no conflicts of interest.

\section{References}

1. Crepeau AZ, Treiman DM. Levetiracetam: a comprehensive review. Expert Rev Neurother 2010;10:159-71.

2. Mbizvo GK, Dixon P, Hutton JL, Marson AG. The adverse effects profile of levetiracetam in epilepsy: a more detailed look. Int I Neurosci 2014; 124:627-34.

3. Krause W. Drug-induced hperpigemntation: a systematic review. J Dtsch Dermatol Ges 2013;11:644-51.

4. Hunter JA, Savin JA, Dahl MV. Disorders of pigmentation. Clinical Dermatology. 3rd ed. Oxford: Blackwell, 2002;242-252.

5. Raj T. Hall JE. The endocrine system. In: Hall JE, ed. Guyton and Hall Textbook of Medical Physiology. A South Asian Edition. New Delhi: Elsevier, 2013;533-622.

6. Halaban R, Hebert D, Fisher D. Biology of Melanocytes. In: Freedberg IM, Eisen AZ, Wolff K, ed. Fitzpatrick's Dermatology in General Medicine. 6th ed. New York: McGraw-Hill; 2003;127-48.

7. Dereure 0. Drug-induced skin pigmentation. Epidemiology, diagnosis and treatment. Am J Clin Dermatol 2001;2:253-62.

8. Garin Shkolnik T, Feuerman H, Didkovsky E, et al. Blue-gray mucocutaneous discoloration: a new adverse effect of ezogabine. JAMA Dermatol 2014;150:984-9.

9. Ramanujam B, Ihtisham K, Kaur G, et al. Spectrum of cutaneous adverse reactions to levetiracetam and human leukocyte antigen typing in North-Indian patients. J Epilepsy Res 2016;6:87-92.

10. Ortonne JP, Passeron T. Melanin pigmentary disorders: treatment update. Dermatol Clin 2005;23:209-26. 